\title{
Carbapenem-Resistant Enterobacteriaceae in Solid Organ Transplantation: Management Principles
}

\author{
Olivia Smibert ${ }^{1,2} \cdot$ Michael J. Satlin ${ }^{3} \cdot$ Anoma Nellore $^{4} \cdot$ Anton Y. Peleg ${ }^{1,5}$ \\ Published online: 10 June 2019 \\ (C) Springer Science+Business Media, LLC, part of Springer Nature 2019
}

\begin{abstract}
Purpose of Review Carbapenem-resistant Enterobacteriaceae (CRE) have emerged as a worldwide problem. Given their degree of immunosuppression and the level of contact with the healthcare system, solid organ transplant (SOT) recipients are at a disproportionately higher risk of acquisition, colonization, and infection with CRE, and outcomes from infection tend to be worse compared to non-transplant patients. Therapeutic options are limited for CRE infections although several newer agents have recently been approved for use. How well these agents perform in the setting of immunosuppression and SOT is unclear. We sought to review the epidemiology of CRE in SOT and the management principles.

Recent Findings CRE infections are becoming an increasing problem in SOT, and donor-derived infections present a challenge in the peri-transplant period. Newer treatments for CRE are emerging that are less toxic and potentially more effective than prior CRE-active agents, but supportive clinical data are limited. Newer beta-lactamase inhibitors have good activity against KPC carbapenemases, but they lack activity against metallo-beta-lactamases (e.g., NDM). Promising data is emerging with newer agents that have activity against most carbapenemases, but, again, clinical data is needed. Combination therapy in addition to optimal pharmacokinetic and pharmacodynamics may go some way to improve outcomes against these difficult-to-treat organisms. Other novel therapies that prevent the emergence of resistance (oral beta-lactamase inhibitors) and eradication of resistant Gram-negative colonization (fecal microbiota transplant) may eventually become part of a bundle approach to reduce CRE infections in the future.

Summary As in non-transplant patients, CRE infections in the transplant setting are challenging to treat and prevent. Infection prevention and control remains crucial to prevent widespread dissemination, and unique challenges exist with donor-derived CRE and how best to manage recipients in the peri-transplant period. Newer treatments are now in early-phase clinical studies, and in vitro activity data are supportive for several agents providing hope for improved outcomes with these typically difficult-totreat and highly morbid infections in transplant recipients.
\end{abstract}

Keywords CRE $\cdot \mathrm{CPE} \cdot$ Transplant $\cdot$ Treatment $\cdot$ Gram-negative bacteria

This article is part of the Topical Collection on Transplant and Oncology

Anton Y. Peleg

Anton.Peleg@monash.edu; anton.peleg@monash.edu

Olivia Smibert

oliviasmibert@alfred.edu.au

Michael J. Satlin

mjs9012@med.cornell.edu

Anoma Nellore

anellore@uabmc.edu

1 Department of Infectious Diseases, The Alfred Hospital and Central Clinical School, Monash University, Melbourne, VIC, Australia
Transplant Infectious Disease and Compromised Host Program, Massachusetts General Hospital, Boston, MA, USA

3 Division of Infectious Diseases, Weill Cornell Medicine, 1300 York Avenue, New York, NY, USA

4 Division of Infectious Diseases, University of Alabama at Birmingham, Birmingham, AL, USA

5 Infection and Immunity Program, Monash Biomedicine Discovery Institute, Department of Microbiology, Monash University, Clayton, VIC, Australia 


\section{Introduction}

It is well-established that infections caused by antimicrobialresistant pathogens represent one of the greatest challenges to human health. Multidrug-resistant Gram-negative bacteria are of most concern, with reports of organisms causing infections that are resistant to all currently available antimicrobials. Carbapenems have been thought of as last-line therapy for multidrug-resistant Gram-negative bacteria, but with the emergence of carbapenemases worldwide, reliance on this antibiotic class is less secure. Patients who are immunocompromised, including solid organ transplant recipients, form one of the highest risk groups for acquisition and infection with carbapenem-resistant Gram-negative bacteria. Solid organ transplantation is an independent risk factor for the development of carbapenem-resistant Enterobacteriaceae (CRE) infection [1]. Transplant recipients have all the established risk factors for colonization and infection with resistant pathogens, such as recurrent exposure to broad-spectrum antimicrobials, extensive healthcare contact, longer length of hospital stays, exposure to the intensive care unit, and renal impairment $[2$, 3]. Infections typically occur early post-transplant, and sites of infection vary by organ transplant type [4]. Lung transplants are associated with pneumonias; liver transplant is associated with intra-abdominal infections and bacteremia; and the urinary tract is the most frequent source in kidney transplant recipients $[5,6]$. Carbapenem resistance is problematic in non-fermenter Gram-negative bacteria, such as Pseudomonas and Acinetobacter, as well as Enterobacteriaceae, such as Klebsiella pneumoniae and Escherichia coli. This review will focus on CRE and its impact on solid organ transplantation, including management principles with regard to donor-derived CRE and newer treatment options of recipients with CRE infection.

\section{Infection Prevention and Control of CRE in Solid Organ Transplantation}

Adequately powered controlled studies are needed to define the best approach for management and control of CRE infection in SOT recipients. No standard guidelines exist for infection prevention in SOT, and centers implement a variety of different strategies [7]. While transmission of MDRO from donors to recipients is well-described, there are only few descriptions of confirmed horizontal transmission among hospitalized SOT recipients [8]. Horizontal transmission is a potentially significant risk, and, therefore, like for other patient groups, best-practice preventative strategies should be established and broadly adopted $[2,5,9,10]$. The majority of the literature represents management of MDRO outbreaks, and eleven studies to date have tested the effect of infection prevention on CRE infection and/or colonization [10-21].
These studies form the basis of an updated guideline for infection prevention of CRE from the World Health Organization [11]. The majority of these studies assessed multi-modal strategies, including contact precautions, patient isolation and surveillance strategies, hand hygiene, antibiotic stewardship, and environmental cleaning. Unfortunately, heterogeneity in the design, intervention, and endpoints in these studies has precluded a comprehensive meta-analysis of pooled results. However, certain insights can be gleaned; six of 11 studies assessed strategies to improve hand hygiene compliance as part of an infection prevention bundle for CRE prevention and 5 of these 6 studies found reduced CRE infection rates with this intervention $[10,12,14,15,17,21]$. Ten of 11 studies assessed the impact of active surveillance for CRE colonization in a combination of high-risk patients and close contacts [10, 12, 14-18, 20, 21]. Screening strategies varied and included longitudinal screening using rectal swabs of patients with known CRE to screening of patients for CRE development, as well as close contacts. Based on results that demonstrated a reduction in incidence of CRE after implementation of screening strategies, the WHO have made a strong recommendation that surveillance should be part of a multimodal strategy to prevent and control CRE health-care associated infections [11]. In addition, the majority of reports demonstrate a reduction in CRE with contact precautions and patient isolation strategies, which similarly form part of the strong recommendations put forward by the WHO. However, the optimal duration of patient isolation or screening postCRE infection or colonization remains unknown, with some groups going so far as to recommend indefinite contact precautions [9]. A minority of studies assessed the role of environmental cleaning with two of three showing a decrease in CRE infection $[10,14,17]$. These studies could not conclude which agents were the best to use for cleaning purposes but did find that education of cleaning staff to be an important aspect of compliance with appropriate environmental cleaning. With respect to transplant recipients, acquisition of CRE from donors is a unique and significant risk, and it has been shown that hospitalization as short as two days is adequate for donor acquisition of a resistant organism that can be transmitted by transplantation $[22,23]$. Communication gaps between facilities had been associated with adverse patient outcomes; therefore, inter-facility communication of CRE infection or colonization forms an essential strategy to reduce acquisition of CRE in these patients [24]. Collectively, these data support the use of multimodal approaches of infection prevention and control to prevent CRE infection and colonization. The relative contribution of any one intervention is difficult to ascertain from the existing literature, and guidelines strongly support a combination of measures [5, 11, 25]. Given the state of the current evidence and the limited number of available studies, it is perhaps not surprising that there are mixed levels of implementation across centers and countries 
when clinicians are surveyed [26]. Significant knowledge gaps regarding best practice remain and include optimal timing and methods of CRE screening, cost-effectiveness of screening in transplant cohorts, and the longitudinal dynamics of CRE colonization and risk factors for extended colonization.

\section{Peri-Transplant Management and Donor-Derived CRE Infections}

Multiple reports have illustrated the potentially devastating consequence of unrecognized transmission of CRE from donor to recipient typically due to the delays in laboratory processing coupled with the often urgent nature of organ procurement from a potential donor [27, 28••, 29-33]. Mularoni et al. described the experience of an Italian centre where $14 \%$ of 214 recipients received an organ from a CRE-infected or colonized donor [32]. Errico et al. demonstrated that 3.4\% of 588 lung and liver donors were colonized with $\mathrm{CRE}$ at the time of transplant [28*0]. Of the recipients, $5 \%$ became CRE-colonized after transplant and occurred exclusively in lung recipients from donors with respiratory tract colonization and 1.9 and $5.3 \%$ of liver and lung recipients, respectively, developed CRE infection. Goldberg et al. described two recipients, each of a single lung from a donor whose colonization with a carbapenem-resistant Klebsiella pneumoniae (CRKP) was identified $48 \mathrm{~h}$ after successful donation, and, despite directed therapy, one of the recipients died of overwhelming and disseminated CRKP bacteremia [31]. Galvano et al. described a heart transplant recipient from a donor found to have colistin-resistant CRKP bacteremia, identified five days after donation. Despite optimization of multi-drug therapy, the patient developed disseminated infection leading to death [29]. As it stands, transplant institutions have varied guidelines about accepting organs from CRE-colonized or infected donors. Physician acceptance varies based on infecting organism, type, or organ being transplanted and, in particular, differentiating between colonization versus infection. A survey of ID Physicians from the American Society of Transplantation Infectious Diseases Community of Practice found that only $10 \%$ of clinicians said they would accept an organ from a donor with CRE bacteremia [34].

These reports highlight the importance of optimal perioperative management where appropriate and directed therapy based on donor cultures is paramount. Mularoni et al. assessed the outcomes of 30 transplant recipients of organs from donors that were either colonized or infected with carbapenemresistant organisms (CRO) [32]. The recipients were divided into low risk, defined as those receiving organs from donors where the CRO infection/colonization was of a nontransplanted organ, and high risk, defined as receiving an organ from a donor with either CRO bacteremia or infection/ colonization of the transplanted organ. Of the 16/30 low-risk recipients, no CRO transmission occurred. Of the 14/30 highrisk recipients, eight received appropriate antibiotic therapy that was started in the first 6 days after transplantation and was continued for $>7$ days. No transmission was reported for these eight patients. The remaining six patients received inappropriate therapy, or therapy was started $>7$ days after transplantation or continued for $<7$ days, and transmission occurred in four of these recipients. In the four recipients where transmission occurred, three developed infection, with one death related to overwhelming sepsis due to the transmitted CRE organism. The reported reasons for the inappropriate or delayed therapy in these six recipients were underestimation of the risk and miscommunication of donor microbiology results.

Optimized communication between organ procurement agencies and transplanting centers must be prioritized to prevent delays in targeted therapy. The decision of whether to transplant an organ from a potential donor who is known to be colonized or infected with CRE requires careful consideration. The risk of transmission appears low for donors that have localized CRE infection or colonization of a nontransplanted organ $[23,32,35]$. The highest risk of transmission is in donors who have CRE bacteremia and infection or colonization of the transplanted organ, such as respiratory tract colonization in a lung donor or gastrointestinal tract colonization in a bowel or pancreas transplant [33]. With the advent of newer and more effective therapies with activity against CRE, as described in subsequent texts, recommendations may become more liberal with regard to accepting such donors.

Although the American Society of Transplantation Infectious Diseases Community of Practice does not routinely recommend antimicrobial therapy for recipients of allografts from donors with non-bacteremic, localized infection of other organs, a careful risk-benefit evaluation in the case of donor colonization or infection with CRE is recommended [36, 37]. A Working Group of the Israeli Society for Infectious Diseases recommended administering at least $48 \mathrm{~h}$ of active targeted therapy to the donor prior to transplantation in this setting, as well as at least three days of targeted therapy to the recipient, starting one hour before the transplant [35]. The Spanish Society of Transplant (SET), the Group for Study of Infection in Transplantation of the Spanish Society of Infectious Diseases and Clinical Microbiology (GESITRA_EIMC), and the Spanish Network for Research in Infectious Diseases (REIPI) recent guidelines for management of CRE in SOT recommend that recipients should receive a minimum of 7 days of effective antibiotics posttransplant and that consideration should be given to avoiding kidney donation from a patient with a CRE-related UTI or lung donation from a patient with CRE-related lower respiratory tract infection or any patient with a CRE bacteremia 
entirely [5]. Given reports of CRE infections occurring in recipients of organs from donors who were thought to only have localized infections of other organs, prophylactic targeted therapy to the recipient and donor, where feasible, is likely prudent [38].

\section{New Antibiotics to Treat CRE Infections}

Prospective clinical trials are still lacking to guide best practice in treatment of CRE infections. Established treatments often include polymyxins, tigecycline, aminoglycosides, and fosfomycin, often in combination; but, here, we will cover the newer antibiotic options.

\section{Ceftazidime-Avibactam}

The addition of avibactam, a diazabicyclooctane $\beta$-lactamase inhibitor, to ceftazidime improves the inhibitory activity of ceftazidime towards many resistant Gram-negative bacteria, including those producing classes $\mathrm{A}$ and $\mathrm{C}$ and some class $\mathrm{D}$ $\beta$-lactamases, including Klebsiella pneumoniae carbapenemases (KPCs) [39-44]. Unfortunately, ceftazidime-avibactam lacks meaningful activity against any of the class B metallo-beta-lactamases (MBLs) [43]. Castanheira et al. demonstrated that $99.3 \%$ of 456 Enterobacteriaceae isolates carrying $b l a_{\mathrm{KPC}}$ collected across US hospitals were susceptible to ceftazidime-avibactam, while de Jonge et al. similarly found $98.7 \%$ susceptibility in 609 carbapenem-resistant, non-MBL-producing isolates, the majority of which were KPC producing [40, 44]. Ceftazidime-avibactam is currently approved by the U.S. Food and Drug Administration (FDA) for the treatment of complicated urinary tract and intra-abdominal infections and hospital-acquired and ventilator-associated pneumonia.

Although limited to case reports and small series, the data supporting ceftazidime-avibactam efficacy in multidrugresistant (MDR) Gram-negative infections in solid organ transplant recipients is accumulating [45]. Jacobs et al. detailed the case of a 47-year-old female who was administered ceftazidime-avibactam as part of a triple drug regimen for the treatment of a KPC3-producing K. pneumoniae intraabdominal abscess and bacteremia post-kidney-pancreas transplant [46]. The patient ultimately succumbed to complications but successfully cleared the bacteremia while on combination therapy. Carmargo et al. described a 64-year-old female with KPC-producing $K$. pneumoniae successfully treated with ceftazidime-avibactam after multiple lines of therapy failed to resolve a peri-pancreatic collection after small bowel transplant [47]. Caravaca-Fontan et al. detailed a patient with recurrent KPC-producing $K$. pneumoniae urinary tract infection after kidney transplant successfully cured of recurrence after treatment with ceftazidime-avibactam [48]. In terms of larger case series, Shields et al. reported a single-center retrospective experience of 109 patients with carbapenem-resistant K. pneumoniae bacteremia (97\% bla $\left._{\mathrm{KPC}}\right)$, with $40(36.7 \%)$ being solid organ transplant recipients [49]. They reported that the administration of ceftazidime-avibactam, either as monotherapy or as part of combination regimen, was associated significantly with clinical success and survival compared to alternative regimens. In an Italian series, Tumbarello et al. reviewed all cases of infections due to KPC-producing K. pneumoniae infection treated with ceftazidime-avibactam as salvage therapy where 35 of 138 patients were organ transplant recipients [50]. The majority of these patients were bacteremic; one third occurred in the ICU and all received ceftazidime-avibactam as part of a multidrug salvage regimen. In this cohort, treatment with ceftazidime-avibactam either as monotherapy or as part of a multidrug regimen was independently associated with survival compared to historical controls.

Emergence of resistance has been described in recipients of SOT receiving ceftazidime-avibactam $[51,52]$. While wildtype $b l a_{\mathrm{KPC}-3}$ and mutations in outer membrane porins have been described in sporadic cases of resistance, prolonged therapy with ceftazidime-avibactam has been linked to mutations in the $b l a_{\mathrm{KPC}-3}$ gene $[51,53]$. For this reason, the authors would recommend ensuring in vitro susceptibility has been determined prior to empiric therapy and consideration be given to repeat testing if infection seems to respond poorly to therapy. Other risk factors for development of resistance, including in SOT recipients, include obesity, renal replacement therapy, and treatment of pneumonia $[41,51,52,54,55]$. In general, ceftazidime-avibactam has been associated with lower rates of toxicity and adverse effects compared to comparator agents, including aminoglycosides, colistin, and tigecycline [56]. No specific risks to solid organ transplant recipients have been identified, and the body of evidence, albeit limited, supports the efficacy of this agent in this patient population as part of either single or combination therapy. Further work defining the pharmacokinetics/pharmacodynamics and optimized dosing of ceftazidime-avibactam in organ transplant recipients would still be welcomed [57-59].

\section{Meropenem-Vaborbactam}

Vaborbactam is a cyclic boronic acid pharmacophore $\beta$ lactamase inhibitor that has demonstrated potent in vitro inhibition of class $\mathrm{A}$ and $\mathrm{C}$ enzymes that, in combination, has increased the potency of meropenem $>64$-fold $[60,61]$. Meropenem-vaborbactam has no activity against MBLs or oxacillinases, but, it appears to have potent inhibitory activity against KPC-producing Enterobacteriaceae [60-62]. Meropenem-vaborbactam has been shown to have lower minimum inhibitory concentrations (MICs) than ceftazidimeavibactam against KPC-producing strains, and less than $25 \%$ 
of ceftazidime-avibactam-resistant strains are resistant to meropenem-vaborbactam [61]. While ceftazidimeavibactam resistance has been described in $b l a_{\mathrm{KPC}-3}$-producing organisms, this vulnerability does not appear to exist for meropenem-vaborbactam [61, 63]. Meropenemvaborbactam has received FDA approval for the treatment of complicated urinary tract infection and acute pyelonephritis based on a large, phase 3 clinical trial that demonstrated non-inferiority to piperacillin-tazobactam for these indications [64]. TANGO II, an open-label randomized control trial comparing meropenem-vaborbactam to best available therapy for known or suspected CRE infection, showed that patients randomized to meropenem-vaborbactam had increased clinical cure and trends towards decreased nephrotoxicity and mortality [65]. This trial was terminated prematurely by the Data Safety Monitoring Board, who deemed that randomization to the best available therapy arm would not be in the best interest of study participants. This trial did not exclude solid organ transplant recipients and analyzed 19 immunocompromised patients, although the exact number of solid organ transplant recipients was not reported.

\section{Imipenem-Relebactam}

Relebactam is a novel $\beta$-lactamase inhibitor, structurally similar to avibactam, with activity against class A (including KPC-type carbapenemases) and C $\beta$-lactamases. When combined with imipenem/cilastatin, relebactam significantly improves the spectrum of activity, reducing the MIC by up to 128-fold, including for isolates producing KPC enzymes [62, 66-68]. While early-phase data suggests that imipenemrelebactam is safe and well-tolerated, phase III clinical data is currently lacking [69].

\section{Aztreonam-Avibactam}

As described previously, the new beta-lactamase inhibitors are welcome news for the treatment of class A carbapenemases, such as KPC, but all are limited by their lack of activity against MBL enzymes. While KPC carbapenemases predominate globally, the prevalence of the more difficult to treat MBLproducing organisms is increasing. These carbapenemases render almost all antibiotics ineffective; however, when other beta-lactamases are not present, MBLs remain susceptible to the monobactam, aztreonam. This led to the innovative combination of aztreonam-avibactam. The avibactam can inhibit other class A, C, and some D beta-lactamases, which allows the aztreonam to be active against the MBL. Like other newer $\beta$-lactam/ $\beta$-lactamase inhibitor combinations, significant aztreonam-avibactam potency towards MBL-negative Enterobacteriaceae has been demonstrated in vitro [70-72]. More excitingly, however, is that this combination has demonstrated improved activity against difficult-to-treat MBL- producing organisms, including IMP-, VIM-, and NDMtype MBLs $[70,73,74]$. Chew et al. showed that the addition of avibactam to aztreonam restored susceptibility of aztreonam in $98.6 \%$ of 70 carbapenem-resistant, dualcarbapenemase-producing (including MBL-producing) Enterobacteriaceae [73]. Marshall et al. demonstrated a significant reduction in ceftazidime/avibactam MIC in 21 ceftazid ime/avibactam-resistant, MBL-producing Enterobacteriaceae with the addition of aztreonam to a multidrug regimen [75]. Shaw et al. recently detailed the experience of treating 10 patients, four of whom were solid organ transplant recipients, with NDM-1-producing $K$. pneumoniae as part of an outbreak with the combination of ceftazidime/ avibactam and aztreonam [76•]. Evidence of synergy was demonstrated in vitro prior to therapy, and treatment was associated with a $60 \%$ success rate without adverse effects.

\section{Other Agents}

Early-phase studies have now been completed for several other new agents with promise against CRE infections; however, the inclusion of solid organ transplant recipients in these studies is limited. These include the new aminoglycoside, plazomicin, which is not degraded by the aminoglycoside-modifying enzymes and therefore has a greater spectrum of activity [77]. It is important to note however that plazomicin is not active against organisms that have a ribosomal methylase, which confers resistance to all aminoglycosides [78]. Ribosomal methylases have often been reported with NDM-type MBL-producing isolates [79]. The CARE trial, a randomized comparison of plazomicin to colistin as part of a multidrug regimen for CRE infections (where the majority were blood-stream infections), recruited a small number of patients but found that plazomycin was associated with improved survival and microbiological clearance compared to colistin [80]. Despite this, recruitment was difficult and small numbers of patients were enrolled, limiting the study's statistical power. There is limited experience in transplant recipients, and nephrotoxicity remains a concern [81]. Another agent that is a modification of an existing class is the tetracycline derivative, eravacycline. Eravacycline is a modified tigecycline that can be administered orally or intravenously and has activity against CRE pathogens with a 1-2-fold lower MIC than tigecycline against these organisms [82]. Finally, a new and exciting agent in the pipeline is a siderophore cephalosporin known as cefiderocol. This antibiotic exploits the active iron uptake system of bacteria to reach its target and appears to have activity against $\mathrm{KPC}$, OXA, and MBL-producing Enterobacteriaceae [83, 84]. We eagerly await clinical studies involving patients, and in particular transplant recipients, with CRE infections. 


\section{Combination Antimicrobial Therapy for CRE Infections}

Combination antibiotic therapy has been associated with improved clinical and microbiological outcomes when treating KPC Enterobacteriaceae in patients with pneumonia and severe sepsis [85-89]. However, robust prospective clinical trial data have been limited, with recommendations based on retrospective series with significant clinical heterogeneity. Results of the AIDA trial, an open-label randomized trial comparing colistin monotherapy to colistin and infusional meropenem for the treatment of carbapenem-resistant, colistin-susceptible Gram-negative infections, were recently presented in the Lancet [90•]. In this cohort of 406 patients, combination therapy was not found to be superior to monotherapy. However, the majority of infections were caused by Acinetobacter baumannii, and there was a lack of therapeutic drug monitoring to ensure $\mathrm{PK} / \mathrm{PD}$ targets were met. While numbers were too few to reach significance, there was a trend to fewer microbiological failures and deaths in those with Enterobacteriaceae infections who received combination therapy. Plans to compile results from the similarly designed Trial for the Treatment of Extensively Drug-Resistant Gramnegative Bacilli (NCT01597973) after completion of enrolment are described by the authors in an attempt to improve the power to detect a difference in the Enterobacteriaceae subgroup. While robust clinical data is currently lacking, evidence for antibiotic synergy has consistently been demonstrated in vitro for a wide range of combinations, including the newer agents described previously [66, 74, 75, 91-93]. Further studies evaluating the efficacy of combination therapy for CRE infections, including in transplant recipients, would be greatly welcomed.

\section{Antimicrobial Pharmacokinetics Relevant to Solid Organ Transplant Recipients}

Solid organ transplant recipients are vulnerable to sepsis and critical illness, increasing their risk for altered pharmacokinetics (PK) and antimicrobial failure [94-96]. $\beta$-lactams are hydrophilic, making them susceptible to the physiological effects of such critical illness, which include alterations in the volume of distribution (Vd) secondary to fluid resuscitation, perturbations in serum albumin, and capillary permeability [97-99]. Similarly, renal drug clearance may either be augmented or reduced, leading to either an increase or decrease in $\beta$-lactam clearance, respectively [97-99]. The efficacy of $\beta$ lactams is proportional to the time non-protein-bound drug spends above the MIC, with maximal bacterial killing and clinical efficacy occurring at target concentrations $4 \times$ MIC $[100,101]$. However, these correlations are based on animal models that used susceptible bacteria [99]. This work did not account for rising antimicrobial MICs and the profound physiological alterations that occur during critical illness and transplantation. Dose optimization of $\beta$-lactams via extended and continuous infusions has been an active area of research to assess their impact on patient outcomes; however, solid organ transplant recipients only form a small sub-group of these studies [95, 101-103].

Transplant recipients often require organ supports, including renal replacement therapy and extracorporeal membrane oxygenation (ECMO), both of which are associated with significant perturbations in the PK of $\beta$-lactam and other antibiotic classes. The circuitry of ECMO has been associated with antibiotic extraction, particularly affecting lipophilic and protein-bound drugs, while hemodilution increases the volume of distribution. However, the extent to which these changes affect $\beta$-lactam PK is less clear [104-106]. While limited evidence suggests that meropenem may achieve more favorable PK compared to piperacillin/tazobactam in the setting of ECMO, data to guide optimal therapy with any of the newer agents is scarce [104, 107].

Barriers to optimal antimicrobial PK vary between organs. For example, recipients undergoing lung transplant for cystic fibrosis (CF) experience significantly increased renal clearance compared to healthy controls [108]. Infection in the lung allografts also presents a significant PK barrier. Penetration of $\beta$-lactams into the pulmonary epithelial lining fluids (ELF) varies dramatically, with ceftazidime, meropenem, piperacillin, and imipenem reported to be $20,30,50$, and $55 \%$, respectively $[99,101,109,110]$. Regarding the newer antimicrobials, ELF/plasma area under the curve (AUC) ratios improve when meropenem is combined with vaborbactam and when ceftazidime is combined with avibactam, while the ELF/ plasma AUC ratio for ceftolozane/tazobactam appears to be about $48 \%[69,111,112]$. Imipenem/cilastatin and relebactam have both been shown to have similar ELF/plasma AUC ratios of around of $55 \%$. The penetrative potency into the pulmonary parenchyma in addition to strong anti-pseudomonal activity support these new agents as potentially important options for patients with difficult-to-treat pseudomonal respiratory tract infections [110].

In liver transplantation, significant physiological perturbations occur early post-transplant that influence antimicrobial PK, including rapid improvement in hepatic metabolic and synthetic function, volume status, and hemodynamics, particularly in cirrhotics [113]. Hepatic metabolic function continues to improve over time, including a normalization of albumin and restoration of hepatic allograft blood flow leading to a subsequent increase in hepatic drug clearance [114]. After heart transplant, changes in the cardiac index and stroke volume occur, theoretically increasing hepatic and renal blood flow. However, the real time effects of antibiotic drug clearance have not been quantified [114]. Early post-kidney transplant, cardiac index decreases, the effect of which is further exacerbated if an arteriovenous fistula remains in situ [115]. 
Despite significant physiological changes occurring in each organ type early post-transplant, the effects of antimicrobial PK in non-critically ill or septic organ transplants have not been determined. Further work is needed to clarify the differences that may compromise optimal therapeutic attainment and represent opportunities for organ-specific intervention. Furthermore, as laboratory resources and capacities expand to facilitate and support real-time therapeutic drug monitoring of antimicrobials, this will likely become a more integral component of antibiotic therapy, particularly for transplant recipients.

\section{Future Perspectives}

Colonization with CRE, which is a risk factor for subsequent invasive infection plus transmission to other patients, predominately occurs within the gut, and it would appear that gut dysbiosis, or disruption of the gut microbiome, increases the risk for colonization with multidrug-resistant bacteria [98, $116,117]$. In essence, a healthy gut microbiome can act to resist colonization with antimicrobial-resistant pathogens, also termed "colonization resistance." [118] Disruption to the gut microbiome is common in transplantation due to recurrent exposures to antimicrobials, immunosuppression, and repeated contact with healthcare $[119,120]$. This leads to the idea of gut microbiome protection or restoration as preventative strategies against gut dysbiosis.

In terms of gut microbiome protection, an exciting and novel approach has emerged with the advent of orally administered recombinant $\beta$-lactamase enzymes. Fifty percent of transplant recipients will experience a bacterial infection post-transplant with many receiving broad-spectrum antibiotics during the peri-transplant period, leading to antibioticinduced dysbiosis and promotion of resistant bacterial overgrowth [121-123]. Recombinant oral $\beta$-lactamase enzymes have been developed that hydrolyze penicillins and cephalosporins but are not systemically absorbed and therefore only function within the gut $[123,124]$. These agents are designed to be administered concurrently with intravenous betalactams, to protect the gut microbiome from the effects of systemic antibiotics. While in their infancy, these therapies present a particularly attractive strategy in transplant recipients who experience multiple infections requiring multiple courses of antimicrobials [124, 125, 126•].

In terms of gut microbiome restoration, microbiomemodulating therapy has been reported on to improve "colonization resistance" against drug-resistant bacteria. Wong et al. undertook a pooled analysis from 101 patients (18 studies) that underwent fecal microbiota transplantation (FMT) and found that $82 \%$ achieved decontamination or significant reduction in carriage of multidrug-resistant bacteria [127]. Based on favorable results with FMT in patients with
C. difficile, interest in microbiome-modulating therapies is increasing [128-131]. As it stands, there is insufficient evidence to recommend FMT in transplant recipients as a strategy to either reduce carriage with multidrug-resistant bacteria or risk of subsequent infection, and there are significant theoretical risks in transplanting fecal flora into the immunocompromised. A number of trials are underway to examine whether carriage of MDR organisms in the gut can be reversed in hospitalized patients, including in solid organ transplantation (NCT02816437), the results of which are highly anticipated.

Acknowledgements Anton Y. Peleg acknowledges support from an Australian National Health and Medical Research Council Practitioner Fellowship.

\section{Compliance with Ethical Standards}

Conflict of Interest Olivia Smibert and Anoma Nellore declare that they have no conflict of interest.

Michael Satlin consulted for Shionogi and Achaogen and has received grant funding from Allergan and Merck. Anton Peleg has received research grant support through the investigator initiated research grant program from MSD.

Human and Animal Rights and Informed Consent This article does not contain any studies with human or animal subjects performed by any of the authors.

\section{References}

Papers of particular interest, published recently, have been highlighted as:

- Of importance

- Of major importance

1. Patel G, Huprikar S, Factor SH, Jenkins SG, Calfee DP. Outcomes of carbapenem-resistant Klebsiella pneumoniae infection and the impact of antimicrobial and adjunctive therapies. Infect Control Hosp Epidemiol. 2008;29(12):1099-106.

2. Moreno Camacho A, Ruiz Camps I. Nosocomial infection in patients receiving a solid organ transplant or haematopoietic stem cell transplant. Enferm Infecc Microbiol Clin. 2014;32(6):386-95.

3. Schwaber MJ, Klarfeld-Lidji S, Navon-Venezia S, Schwartz D, Leavitt A, Carmeli Y. Predictors of carbapenem-resistant Klebsiella pneumoniae acquisition among hospitalized adults and effect of acquisition on mortality. Antimicrob Agents Chemother. 2008;52(3):1028-33.

4. Lanini S, Costa AN, Puro V, Procaccio F, Grossi PA, Vespasiano $\mathrm{F}$, et al. Incidence of carbapenem-resistant gram negatives in Italian transplant recipients: a nationwide surveillance study. PLoS One. 2015;10(4):e0123706.

5. Aguado JM, Silva JT, Fernandez-Ruiz M, et al. Management of multidrug resistant Gram-negative bacilli infections in solid organ transplant recipients: SET/GESITRA-SEIMC/REIPI recommendations. Transplantation Reviews (Orlando, Fla). 2018;32(1):3657.

6. Satlin MJ, Jenkins SG, Walsh TJ. The global challenge of carbapenem-resistant Enterobacteriaceae in transplant recipients 
and patients with hematologic malignancies. Clinical Infectious Diseases: An Official Publication of the Infectious Diseases Society of America. 2014;58(9):1274-83.

7. Beam E, Razonable RR. A survey of infection prevention and control practices among solid organ transplantation centers. Am J Infect Control 2018.

8. Mathers AJ, Cox HL, Bonatti H, Kitchel B, Brassinga AKC, Wispelwey B, et al. Fatal cross infection by carbapenemresistant Klebsiella in two liver transplant recipients. Transplant Infectious Disease: An Official Journal of the Transplantation Society. 2009;11(3):257-65.

9. Banach DB, Bearman G, Barnden M, Hanrahan JA, Leekha S, Morgan DJ, et al. Duration of contact precautions for acute-care settings. Infect Control Hosp Epidemiol. 2018;39(2):127-44.

10. Viale P, Tumietto F, Giannella M, Bartoletti M, Tedeschi S, Ambretti S, et al. Impact of a hospital-wide multifaceted programme for reducing carbapenem-resistant Enterobacteriaceae infections in a large teaching hospital in northern Italy. Clinical Microbiology and Infection: The Official Publication of the European Society of Clinical Microbiology and Infectious Diseases. 2015;21(3):242-7.

11. WHO Guidelines Approved by the Guidelines Review Committee. In: Guidelines for the prevention and control of carbapenem-resistant Enterobacteriaceae, Acinetobacter baumannii and Pseudomonas aeruginosa in health care facilities. Geneva: World Health Organization. Copyright (c) World Health Organization 2017; 2017.

12. Ben-David D, Masarwa S, Adler A, Mishali H, Carmeli Y, Schwaber MJ. A national intervention to prevent the spread of carbapenem-resistant Enterobacteriaceae in Israeli post-acute care hospitals. Infect Control Hosp Epidemiol. 2014;35(7):802-9.

13. Campos AC, Albiero J, Ecker AB, Kuroda CM, Meirelles LEF, Polato A, et al. Outbreak of Klebsiella pneumoniae carbapenemase-producing K pneumoniae: a systematic review. Am J Infect Control. 2016;44(11):1374-80.

14. Ciobotaro P, Oved M, Nadir E, Bardenstein R, Zimhony O. An effective intervention to limit the spread of an epidemic carbapenem-resistant Klebsiella pneumoniae strain in an acute care setting: from theory to practice. Am J Infect Control. 2011;39(8):671-7.

15. DalBen MF, Teixeira Mendes E, Moura ML, et al. A model-based strategy to control the spread of carbapenem-resistant Enterobacteriaceae: simulate and implement. Infect Control Hosp Epidemiol. 2016;37(11):1315-22.

16. Enfield KB, Huq NN, Gosseling MF, Low DJ, Hazen KC, Toney DM, et al. Control of simultaneous outbreaks of carbapenemaseproducing Enterobacteriaceae and extensively drug-resistant Acinetobacter baumannii infection in an intensive care unit using interventions promoted in the Centers for Disease Control and Prevention 2012 carbapenemase-resistant Enterobacteriaceae toolkit. Infect Control Hosp Epidemiol. 2014;35(7):810-7.

17. Gagliotti C, Cappelli V, Carretto E, et al. Control of carbapenemase-producing Klebsiella pneumoniae: a regionwide intervention. Euro Surveillance: Bulletin Europeen sur les maladies transmissibles $=$ European Communicable Disease Bulletin. 2014;19:43.

18. Hayden MK, Lin MY, Lolans K, Weiner S, Blom D, Moore NM, et al. Prevention of colonization and infection by Klebsiella pneumoniae carbapenemase-producing Enterobacteriaceae in long-term acute-care hospitals. Clinical Infectious Diseases: An Official Publication of the Infectious Diseases Society of America. 2015;60(8):1153-61.

19. Kim NH, Han WD, Song KH, Seo HK, Shin MJ, Kim TS, et al. Successful containment of carbapenem-resistant Enterobacteriaceae by strict contact precautions without active surveillance. Am J Infect Control. 2014;42(12):1270-3.
20. Schwaber MJ, Lev B, Israeli A, Solter E, Smollan G, Rubinovitch $\mathrm{B}$, et al. Containment of a country-wide outbreak of carbapenemresistant Klebsiella pneumoniae in Israeli hospitals via a nationally implemented intervention. Clinical Infectious Diseases: An Official Publication of the Infectious Diseases Society of America. 2011;52(7):848-55.

21. Borer A, Eskira S, Nativ R, Saidel-Odes L, Riesenberg K, LivshizRiven I, et al. A multifaceted intervention strategy for eradication of a hospital-wide outbreak caused by carbapenem-resistant Klebsiella pneumoniae in Southern Israel. Infect Control Hosp Epidemiol. 2011;32(12):1158-65.

22. Martins N, Martins IS, de Freitas WV, et al. Severe infection in a lung transplant recipient caused by donor-transmitted carbapenem-resistant Acinetobacter baumannii. Transplant Infectious Disease: An Official Journal of the Transplantation Society. 2012;14(3):316-20.

23. Fishman JA, Grossi PA. Donor-derived infection - the challenge for transplant safety. Nat Rev Nephrol. 2014;10(11):663-72.

24. Miller R, Covington S, Taranto S, Carrico R, Ehsan A, Friedman $\mathrm{B}$, et al. Communication gaps associated with donor-derived infections. Am J Transplant Off J Am Soc Transplant Am Soc Transplant Surg. 2015;15(1):259-64.

25. Tacconelli E, Cataldo MA, Dancer SJ, et al. ESCMID guidelines for the management of the infection control measures to reduce transmission of multidrug-resistant Gram-negative bacteria in hospitalized patients. Clinical Microbiology and Infection: The Official Publication of the European Society of Clinical Microbiology and Infectious Diseases. 2014;20 Suppl 1:1-55.

26. Gysin DV, Cookson B, Saenz H, Dettenkofer M, Widmer AF. Variability in contact precautions to control the nosocomial spread of multi-drug resistant organisms in the endemic setting: a multinational cross-sectional survey. Antimicrob Resist Infect Control. 2018;7:81.

27. Ariza-Heredia EJ, Patel R, Blumberg EA, Walker RC, Lewis R, Evans $J$, et al. Outcomes of transplantation using organs from a donor infected with Klebsiella pneumoniae carbapenemase (KPC)-producing K. pneumoniae. Transplant Infectious Disease: An Official Journal of the Transplantation Society. 2012;14(3): 229-36.

28.• Errico G, Gagliotti C, Monaco M, et al. Colonization and infection due to carbapenemase-producing Enterobacteriaceae in liver and lung transplant recipients and donor-derived transmission: a prospective cohort study conducted in Italy. Clinical Microbiology and Infection: The Official Publication of the European Society of Clinical Microbiology and Infectious Diseases. 2018. Important study that described the experience of donorderived CPE across multiple Italian transplant centres, and documented the outcomes of the recipients.

29. Galvao LM, Oliveira APR, Ibanes AS, et al. Fatal case of donorderived colistin-resistant carbapenemase-producing Klebsiella pneumoniae transmission in cardiac transplantation. The Brazilian Journal of Infectious Diseases: An Official Publication of the Brazilian Society of Infectious Diseases. 2018;22(3):235-8.

30. Giani T, Conte V, Mandala S, D'Andrea MM, Luzzaro F, Conaldi PG, et al. Cross-infection of solid organ transplant recipients by a multidrug-resistant Klebsiella pneumoniae isolate producing the OXA-48 carbapenemase, likely derived from a multiorgan donor. J Clin Microbiol. 2014;52(7):2702-5.

31. Goldberg E, Bishara J, Lev S, Singer P, Cohen J. Organ transplantation from a donor colonized with a multidrug-resistant organism: a case report. Transplant Infectious Disease: An Official Journal of the Transplantation Society. 2012;14(3):296-9.

32. Mularoni A, Bertani A, Vizzini G, Gona F, Campanella M, Spada $\mathrm{M}$, et al. Outcome of transplantation using organs from donors infected or colonized with carbapenem-resistant gram-negative 
bacteria. Am J Transplant Off J Am Soc Transplant Am Soc Transplant Surg. 2015;15(10):2674-82.

33. Varotti G, Dodi F, Marchese A, Terulla A, Bertocchi M, Fontana I. Fatal donor-derived carbapenem-resistant Klebsiella pneumoniae infection in a combined kidney-pancreas transplantation. Case Reports in Transplantation. 2016;2016:7920951.

34. Mehta SR, Logan C, Kotton CN, Kumar D, Aslam S. Use of organs from donors with bloodstream infection, pneumonia, and influenza: results of a survey of infectious diseases practitioners. Transplant Infectious Disease: An Official Journal of the Transplantation Society. 2017;19(1).

35. Bishara J, Goldberg E, Lev S, Singer P, Ashkenazi T, Cohen J. The utilization of solid organs for transplantation in the setting of infection with multidrug-resistant organisms: an expert opinion. Clin Transpl. 2012;26(6):811-5.

36. Fischer SA, Lu K. Screening of donor and recipient in solid organ transplantation. Am J Transplant Off J Am Soc Transplant Am Soc Transplant Surg. 2013;13(Suppl 4):9-21.

37. van Duin D, van Delden C. Multidrug-resistant gram-negative bacteria infections in solid organ transplantation. Am J Transplant Off J Am Soc Transplant Am Soc Transplant Surg. 2013;13 Suppl 4:31-41.

38. Mills JP, Wilck MB, Weikert BC, Porrett PM, Timko D, Alby K, et al. Successful treatment of a disseminated infection with extensively drug-resistant Klebsiella pneumoniae in a liver transplant recipient with a fosfomycin-based multidrug regimen. Transplant Infectious Disease: An Official Journal of the Transplantation Society. 2016;18(5):777-81.

39. Alatoom A, Elsayed H, Lawlor K, et al. Comparison of antimicrobial activity between ceftolozane-tazobactam and ceftazidimeavibactam against multidrug-resistant isolates of Escherichia coli, Klebsiella pneumoniae, and Pseudomonas aeruginosa. International Journal of Infectious Diseases: IJID: Official Publication of the International Society for Infectious Diseases. 2017;62:39-43.

40. de Jonge BL, Karlowsky JA, Kazmierczak KM, Biedenbach DJ, Sahm DF, Nichols WW. In vitro susceptibility to ceftazidimeavibactam of carbapenem-nonsusceptible Enterobacteriaceae isolates collected during the INFORM Global Surveillance Study (2012 to 2014). Antimicrob Agents Chemother. 2016;60(5): 3163-9.

41. Haidar G, Clancy CJ, Chen L, Samanta P, Shields RK, Kreiswirth $\mathrm{BN}$, Nguyen MH Identifying spectra of activity and therapeutic niches for ceftazidime-avibactam and imipenem-relebactam against carbapenem-resistant Enterobacteriaceae. Antimicrob Agents Chemother 2017;61(9).

42. Sader HS, Mendes RE, Pfaller MA, Shortridge D, Flamm RK, Castanheira M. Antimicrobial activities of aztreonam-avibactam and comparator agents against contemporary (2016) clinical Enterobacteriaceae isolates. Antimicrob Agents Chemother 2018;62(1)

43. Carvalhaes CG, Ramos AC, Oliveira LCG, Juliano MA, Gales AC. Rapid detection of ceftazidime/avibactam resistance by MALDI-TOF MS. J Antimicrob Chemother. 2018;73:2579-82.

44. Castanheira M, Mendes RE, Sader HS. Low frequency of ceftazidime-avibactam resistance among Enterobacteriaceae isolates carrying blaKPC collected in U.S. hospitals from 2012 to 2015. Antimicrob Agents Chemother 2017;61(3).

45. Temkin E, Torre-Cisneros J, Beovic B, et al. Ceftazidimeavibactam as salvage therapy for infections caused by carbapenem-resistant organisms. Antimicrob Agents Chemother $2017 ; 61(2)$

46. Jacobs DM, DiTursi S, Ruh C, Sharma R, Claus J, Banjade R, et al. Combination treatment with extended-infusion ceftazidime/ avibactam for a KPC-3-producing Klebsiella pneumoniae bacteraemia in a kidney and pancreas transplant patient. Int $\mathrm{J}$ Antimicrob Agents. 2016;48(2):225-7.

47. Camargo JF, Simkins J, Beduschi T, Tekin A, Aragon L, PérezCardona A, et al. Successful treatment of carbapenemaseproducing pandrug-resistant Klebsiella pneumoniae bacteremia. Antimicrob Agents Chemother. 2015;59(10):5903-8.

48. Caravaca-Fontan F, Jimenez-Alvaro S, Marcen-Letosa R, Fernandez-Rodriguez A, Rodriguez-Navarro CQ. Ceftazidimeavibactam in urinary tract infections due to carbapenemaseproducing Klebsiella in kidney transplantation. Nefrologia: publicacion oficial de la Sociedad Espanola Nefrologia. 2015;35(4):412-3.

49. Shields RK, Nguyen MH, Chen L, Press EG, Potoski BA, Marini $\mathrm{RV}$, et al. Ceftazidime-avibactam is superior to other treatment regimens against carbapenem-resistant Klebsiella pneumoniae bacteremia. Antimicrob Agents Chemother. 2017;61(8).

50. Tumbarello M, Trecarichi EM, Corona A, et al. Efficacy of ceftazidime-avibactam salvage therapy in patients with infections caused by KPC-producing Klebsiella pneumoniae. Clinical Infectious Diseases: An Official Publication of the Infectious Diseases Society of America. 2018.

51. Shields RK, Chen L, Cheng S, et al. Emergence of ceftazidimeavibactam resistance due to plasmid-borne blaKPC-3 mutations during treatment of carbapenem-resistant Klebsiella pneumoniae infections. Antimicrob Agents Chemother 2017;61(3).

52. Wenzler E, Bunnell KL, Bleasdale SC, Benken S, Danziger LH, Rodvold KA. Pharmacokinetics and dialytic clearance of ceftazidime-avibactam in a critically ill patient on continuous venovenous hemofiltration. Antimicrob Agents Chemother 2017;61(7).

53. Shields RK, Nguyen MH, Chen L, Press EG, Kreiswirth BN, Clancy CJ. Pneumonia and renal replacement therapy are risk factors for ceftazidime-avibactam treatment failures and resistance among patients with carbapenem-resistant Enterobacteriaceae infections. Antimicrob Agents Chemother. 2018;62(5):e02497-17.

54. Barber KE, Pogue JM, Warnock HD, Bonomo RA, Kaye KS. Ceftazidime/avibactam versus standard-of-care agents against carbapenem-resistant Enterobacteriaceae harbouring blaKPC in a one-compartment pharmacokinetic/pharmacodynamic model. J Antimicrob Chemother. 2018;73:2405-10.

55. Veillette JJ, Truong J, Forland SC. Pharmacokinetics of ceftazidime-avibactam in two patients with KPC-producing Klebsiella pneumoniae bacteremia and renal impairment. Pharmacotherapy. 2016;36(11):e172-7.

56. van Duin D, Lok JJ, Earley M, Cober E, Richter SS, Perez F, et al. Colistin versus ceftazidime-avibactam in the treatment of infections due to carbapenem-resistant Enterobacteriaceae. Clinical Infectious Diseases: An Official Publication of the Infectious Diseases Society of America. 2018;66(2):163-71.

57. King M, Heil E, Kuriakose S, Bias T, Huang V, el-Beyrouty C, McCoy D, Hiles J, Richards L, Gardner J, Harrington N, Biason K, Gallagher JC Multicenter study of outcomes with ceftazidimeavibactam in patients with carbapenem-resistant Enterobacteriaceae infections. Antimicrob Agents Chemother 2017;61(7).

58. Krapp F, Grant JL, Sutton SH, Ozer EA, Barr VO. Treating complicated carbapenem-resistant Enterobacteriaceae infections with ceftazidime/avibactam: a retrospective study with molecular strain characterisation. Int J Antimicrob Agents. 2017;49(6):770-3.

59. Stone GG, Newell P, Gasink LB, Broadhurst H, Wardman A, Yates K, et al. Clinical activity of ceftazidime/avibactam against MDR Enterobacteriaceae and Pseudomonas aeruginosa: pooled data from the ceftazidime/avibactam phase III clinical trial programme. J Antimicrob Chemother. 2018;73:2519-23.

60. Castanheira M, Rhomberg PR, Flamm RK, Jones RN. Effect of the beta-lactamase inhibitor vaborbactam combined with 
meropenem against serine carbapenemase-producing Enterobacteriaceae. Antimicrob Agents Chemother. 2016;60(9): 5454-8.

61. Hackel MA, Lomovskaya O, Dudley MN, Karlowsky JA, Sahm DF. In vitro activity of meropenem-vaborbactam against clinical isolates of KPC-positive Enterobacteriaceae. Antimicrob Agents Chemother 2018;62(1).

62. Lapuebla A, Abdallah M, Olafisoye O, Cortes C, Urban C, Landman D, et al. Activity of imipenem with relebactam against gram-negative pathogens from New York City. Antimicrob Agents Chemother. 2015;59(8):5029-31.

63. Livermore DM, Warner M, Jamrozy D, Mushtaq S, Nichols WW, Mustafa N, et al. In vitro selection of ceftazidime-avibactam resistance in Enterobacteriaceae with KPC-3 carbapenemase. Antimicrob Agents Chemother. 2015;59(9):5324-30.

64. Kaye KS, Bhowmick T, Metallidis S, Bleasdale SC, Sagan OS, Stus V, et al. Effect of Meropenem-Vaborbactam vs PiperacillinTazobactam on clinical cure or improvement and microbial eradication in complicated urinary tract infection: the TANGO I randomized clinical trial. JAMA. 2018:319(8):788-99.

65. Wunderink RG, Giamarellos-Bourboulis EJ, Rahav G, Mathers AJ, Bassetti M, Vazquez J, et al. Effect and safety of Meropenem-Vaborbactam versus best-available therapy in patients with Carbapenem-resistant Enterobacteriaceae infections: the TANGO II randomized clinical trial. Infect Dis Ther. 2018;7(4):439-55.

66. Livermore DM, Warner M, Mushtaq S. Activity of MK-7655 combined with imipenem against Enterobacteriaceae and Pseudomonas aeruginosa. J Antimicrob Chemother. 2013;68(10):2286-90.

67. Lob SH, Hoban DJ, Young K, Motyl MR, Sahm DF. Activity of Imipenem-Relebactam against Gram-negative bacilli from global ICU and non-ICU wards-SMART 2015-2016. Journal of Global Antimicrobial Resistance. 2018;15:12-9.

68. Zhanel GG, Lawrence CK, Adam H, Schweizer F, Zelenitsky S, Zhanel M, et al. Imipenem-Relebactam and MeropenemVaborbactam: two novel Carbapenem-beta-lactamase inhibitor combinations. Drugs. 2018;78(1):65-98.

69. Nicolau DP, Siew L, Armstrong J, Li J, Edeki T, Learoyd M, et al. Phase 1 study assessing the steady-state concentration of ceftazidime and avibactam in plasma and epithelial lining fluid following two dosing regimens. J Antimicrob Chemother. 2015;70(10): 2862-9.

70. Biedenbach DJ, Kazmierczak K, Bouchillon SK, Sahm DF, Bradford PA. In vitro activity of aztreonam-avibactam against a global collection of Gram-negative pathogens from 2012 and 2013. Antimicrob Agents Chemother. 2015;59(7):4239-48.

71. Karlowsky JA, Kazmierczak KM, de Jonge BLM, Hackel MA, Sahm DF, Bradford PA. In vitro activity of Aztreonam-Avibactam against Enterobacteriaceae and Pseudomonas aeruginosa isolated by clinical laboratories in 40 countries from 2012 to 2015 . Antimicrob Agents Chemother 2017;61(9).

72. Vasoo S, Cunningham SA, Cole NC, Kohner PC, Menon SR, Krause KM, et al. In vitro activities of Ceftazidime-Avibactam, Aztreonam-Avibactam, and a panel of older and contemporary antimicrobial agents against Carbapenemase-producing Gramnegative bacilli. Antimicrob Agents Chemother. 2015;59(12): 7842-6.

73. Chew KL, Tay MKL, Cheng B, Lin RTP, Octavia S, Teo JWP. Aztreonam-Avibactam combination restores susceptibility of Aztreonam in dual-Carbapenemase-producing Enterobacteriaceae. Antimicrob Agents Chemother 2018;62(8).

74. Jayol A, Nordmann P, Poirel L, Dubois V. Ceftazidime/avibactam alone or in combination with aztreonam against colistin-resistant and carbapenemase-producing Klebsiella pneumoniae. J Antimicrob Chemother. 2018;73(2):542-4.
75. Marshall S, Hujer AM, Rojas LJ, Papp-Wallace KM, Humphries RM, Spellberg B, Hujer KM, Marshall EK, Rudin SD, Perez F, Wilson BM, Wasserman RB, Chikowski L, Paterson DL, Vila AJ, van Duin D, Kreiswirth BN, Chambers HF, Fowler Jr VG, Jacobs MR, Pulse ME, Weiss WJ, Bonomo RA Can CeftazidimeAvibactam and Aztreonam overcome beta-lactam resistance conferred by metallo-beta-lactamases in Enterobacteriaceae? Antimicrob Agents Chemother 2017;61(4).

76. Shaw E, Rombauts A, Tubau F, Padullés A, Càmara J, Lozano T, et al. Clinical outcomes after combination treatment with ceftazidime/avibactam and aztreonam for NDM-1/OXA-48/ CTX-M-15-producing Klebsiella pneumoniae infection. J Antimicrob Chemother. 2018;73(4):1104-6. MBL producing Gram negatives are particularly difficult to treat with many of the newer agents providing little to no additional coverage. However, the amalgamation of avibactam with aztreonam presents an exciting combination therapy with evidence of improved efficacy in vitro and in vivo.

77. Cox G, Ejim L, Stogios PJ, Koteva K, Bordeleau E, Evdokimova E, et al. Plazomicin retains antibiotic activity against most aminoglycoside modifying enzymes. ACS Infectious Diseases. 2018;4(6):980-7.

78. Doi Y, Wachino JI, Arakawa Y. Aminoglycoside resistance: the emergence of acquired 16S ribosomal RNA methyltransferases. Infect Dis Clin N Am. 2016;30(2):523-37.

79. Taylor E, Sriskandan S, Woodford N, Hopkins KL. High prevalence of $16 \mathrm{~S}$ rRNA methyltransferases among carbapenemaseproducing Enterobacteriaceae in the UK and Ireland. Int $\mathrm{J}$ Antimicrob Agents. 2018;52(2):278-82.

80. JA McKinnell, Connolly L, Pushkin R, et al. Improved outcomes with Plazomicin (PLZ) compared with Colistin (CST) in patients with bloodstream infections (BSI) caused by carbapenem-resistant Enterobacteriaceae (CRE): results from the CARE study. Vol 42017.

81. Komirenko AS, Riddle V, Gibbons JA, Van Wart S, Seroogy JD. A phase 1 study to assess the pharmacokinetics of intravenous Plazomicin in adult subjects with varying degrees of renal function. Antimicrob Agents Chemother 2018;62(12).

82. Zhanel GG, Cheung D, Adam H, Zelenitsky S, Golden A, Schweizer F, et al. Review of Eravacycline, a novel Fluorocycline antibacterial agent. Drugs. 2016;76(5):567-88.

83. Ito A, Kohira N, Bouchillon SK, West J, Rittenhouse S, Sader HS, et al. In vitro antimicrobial activity of S-649266, a catecholsubstituted siderophore cephalosporin, when tested against nonfermenting Gram-negative bacteria. J Antimicrob Chemother. 2016;71(3):670-7.

84. Kohira N, West J, Ito A, Ito-Horiyama T, Nakamura R, Sato T, et al. In vitro antimicrobial activity of a siderophore cephalosporin, S-649266, against Enterobacteriaceae clinical isolates, including Carbapenem-resistant strains. Antimicrob Agents Chemother. 2016;60(2):729-34

85. Escola-Verge L, Pigrau C, Los-Arcos I, et al. Ceftolozane/ tazobactam for the treatment of XDR Pseudomonas aeruginosa infections. Infection. 2018;46:461-8.

86. Falagas ME, Lourida P, Poulikakos P, Rafailidis PI, Tansarli GS. Antibiotic treatment of infections due to carbapenem-resistant Enterobacteriaceae: systematic evaluation of the available evidence. Antimicrob Agents Chemother. 2014;58(2):654-63.

87. Lee GC, Burgess DS. Treatment of Klebsiella pneumoniae carbapenemase (KPC) infections: a review of published case series and case reports. Ann Clin Microbiol Antimicrob. 2012;11: 32.

88. Parchem NL, Bauer KA, Cook CH, Mangino JE, Jones CD, Porter $\mathrm{K}$, et al. Colistin combination therapy improves microbiologic cure in critically ill patients with multi-drug resistant gramnegative pneumonia. European Journal of Clinical Microbiology 
\& Infectious Diseases: Official Publication of the European Society of Clinical Microbiology. 2016;35(9):1433-9.

89. Pournaras S, Vrioni G, Neou E, Dendrinos J, Dimitroulia E, Poulou A, et al. Activity of tigecycline alone and in combination with colistin and meropenem against Klebsiella pneumoniae carbapenemase (KPC)-producing Enterobacteriaceae strains by time-kill assay. Int J Antimicrob Agents. 2011;37(3):244-7.

90. Paul M, Daikos GL, Durante-Mangoni E, et al. Colistin alone versus colistin plus meropenem for treatment of severe infections caused by carbapenem-resistant Gram-negative bacteria: an openlabel, randomised controlled trial. Lancet Infect Dis. 2018;18(4): 391-400. An important randomised trial that attempted to determine the benefit of combination versus monotherapy for CRE infection. Despite the negative results, the improtant caveat is that this trail was biased to treatment of Acinetobacter infections and results should not be generalised to infections with Enterobacteriaceae.

91. Barlow G, Morice A. Successful treatment of resistant Burkholderia multivorans infection in a patient with cystic fibrosis using ceftazidime/avibactam plus aztreonam. J Antimicrob Chemother. 2018;73(8):2270-1.

92. Monogue ML, Nicolau DP. Antibacterial activity of ceftolozane/ tazobactam alone and in combination with other antimicrobial agents against MDR Pseudomonas aeruginosa. J Antimicrob Chemother. 2018;73(4):942-52.

93. Samonis G, Maraki S, Karageorgopoulos DE, Vouloumanou EK, Falagas ME. Synergy of fosfomycin with carbapenems, colistin, netilmicin, and tigecycline against multidrug-resistant Klebsiella pneumoniae, Escherichia coli, and Pseudomonas aeruginosa clinical isolates. European Journal of Clinical Microbiology \& Infectious Diseases: Official Publication of the European Society of Clinical Microbiology. 2012;31(5):695-701.

94. Donnelly JP, Locke JE, MacLennan PA, et al. Inpatient mortality among solid organ transplant recipients hospitalized for sepsis and severe sepsis. Clinical Infectious Diseases: An Official Publication of the Infectious Diseases Society of America. 2016;63(2):18694.

95. Roberts JA, Abdul-Aziz MH, Davis JS, Dulhunty JM, Cotta MO, Myburgh J, et al. Continuous versus intermittent beta-lactam infusion in severe sepsis. A meta-analysis of individual patient data from randomized trials. Am J Respir Crit Care Med. 2016;194(6): 681-91.

96. Blot SI, Pea F, Lipman J. The effect of pathophysiology on pharmacokinetics in the critically ill patient - concepts appraised by the example of antimicrobial agents. Adv Drug Deliv Rev. 2014;77:3-11.

97. Dhaese SAM, De Kezel M, Callant M, et al. Emergence of antimicrobial resistance to piperacillin/tazobactam or meropenem in the ICU: intermittent versus continuous infusion. A retrospective cohort study. J Crit Care. 2018;47:164-8.

98. Masich AM, Heavner MS, Gonzales JP, Claeys KC. Pharmacokinetic/pharmacodynamic considerations of betalactam antibiotics in adult critically ill patients. Curr Infect Dis Rep. 2018;20(5):9.

99. Osthoff M, Siegemund M, Balestra G, Abdul-Aziz MH, Roberts JA. Prolonged administration of beta-lactam antibiotics - a comprehensive review and critical appraisal. Swiss Med Wkly. 2016;146:w14368.

100. Delattre IK, Taccone FS, Jacobs F, Hites M, Dugernier T, Spapen $\mathrm{H}$, et al. Optimizing beta-lactams treatment in critically-ill patients using pharmacokinetics/pharmacodynamics targets: are first conventional doses effective? Expert Rev Anti-Infect Ther. 2017;15(7):677-88.

101. Roberts JA, Paul SK, Akova M, Bassetti M, de Waele JJ, Dimopoulos G, et al. DALI: defining antibiotic levels in intensive care unit patients: are current beta-lactam antibiotic doses sufficient for critically ill patients? Clinical Infectious Diseases: An Official Publication of the Infectious Diseases Society of America. 2014;58(8):1072-83.

102. Abdul-Aziz MH, Sulaiman H, Mat-Nor MB, Rai V, Wong KK, Hasan MS, et al. Beta-Lactam Infusion in Severe Sepsis (BLISS): a prospective, two-centre, open-labelled randomised controlled trial of continuous versus intermittent beta-lactam infusion in critically ill patients with severe sepsis. Intensive Care Med. 2016;42(10):1535-45.

103. Vardakas KZ, Voulgaris GL, Maliaros A, Samonis G, Falagas ME. Prolonged versus short-term intravenous infusion of antipseudomonal beta-lactams for patients with sepsis: a systematic review and meta-analysis of randomised trials. Lancet Infect Dis. 2018;18(1):108-20.

104. Donadello K, Antonucci E, Cristallini S, Roberts JA, Beumier M, Scolletta S, et al. Beta-lactam pharmacokinetics during extracorporeal membrane oxygenation therapy: a case-control study. Int J Antimicrob Agents. 2015;45(3):278-82.

105. Romano TG, Mendes PV, Park M, Costa EL. Extracorporeal respiratory support in adult patients. Jornal brasileiro de pneumologia: publicacao oficial da Sociedade Brasileira de Pneumologia e Tisilogia. 2017;43(1):60-70.

106. Sherwin J, Heath T, Watt K. Pharmacokinetics and dosing of antiinfective drugs in patients on extracorporeal membrane oxygenation: a review of the current literature. Clin Ther. 2016;38(9): 1976-94.

107. Shekar K, Roberts JA, Ghassabian S, Mullany DV, Wallis SC, Smith MT, et al. Altered antibiotic pharmacokinetics during extracorporeal membrane oxygenation: cause for concern? J Antimicrob Chemother. 2013;68(3):726-7.

108. Rey E, Treluyer JM, Pons G. Drug disposition in cystic fibrosis. Clin Pharmacokinet. 1998;35(4):313-29.

109. Lodise TP, Sorgel F, Melnick D, Mason B, Kinzig M, Drusano GL. Penetration of meropenem into epithelial lining fluid of patients with ventilator-associated pneumonia. Antimicrob Agents Chemother. 2011;55(4):1606-10.

110. Rizk ML, Rhee EG, Jumes PA, Gotfried MH, Zhao T, Mangin E, et al. Intrapulmonary pharmacokinetics of Relebactam, a novel beta-lactamase inhibitor, dosed in combination with ImipenemCilastatin in healthy subjects. Antimicrob Agents Chemother. 2018;62(3).

111. Falcone M, Viale P, Tiseo G, Pai M. Pharmacokinetic drug evaluation of avibactam + ceftazidime for the treatment of hospitalacquired pneumonia. Expert Opin Drug Metab Toxicol. 2018;14(3):331-40.

112. Jorgensen SCJ, Rybak MJ. Meropenem and Vaborbactam: stepping up the battle against Carbapenem-resistant Enterobacteriaceae. Pharmacotherapy. 2018;38(4):444-61.

113. Gerard C, Stocco J, Hulin A, et al. Determination of the most influential sources of variability in tacrolimus trough blood concentrations in adult liver transplant recipients: a bottom-up approach. AAPS J. 2014;16(3):379-91.

114. Greenberg ML, Uretsky BF, Reddy PS, Bernstein RL, Griffith BP, Hardesty RL, et al. Long-term hemodynamic follow-up of cardiac transplant patients treated with cyclosporine and prednisone. Circulation. 1985;71(3):487-94.

115. Czyzewski L, Wyzgal J, Czyzewska E, Sanko-Resmer J, Szarpak L. Assessment of volumetric hemodynamic parameters and body composition in stable renal transplant recipients. Annals of Transplantation. 2017;22:187-98.

116. Holler E, Butzhammer P, Schmid K, Hundsrucker C, Koestler J, Peter K, et al. Metagenomic analysis of the stool microbiome in patients receiving allogeneic stem cell transplantation: loss of diversity is associated with use of systemic antibiotics and more pronounced in gastrointestinal graft-versus-host disease. Biology of Blood and Marrow Transplantation: Journal of the American 
Society for Blood and Marrow Transplantation. 2014;20(5):6405.

117. Muggeo A, Guillard T, Barbe C, Thierry A, Bajolet O, VernetGarnier V, et al. Factors associated with carriage of carbapenemnon-susceptible Enterobacteriaceae in North-Eastern France and outcomes of infected patients. J Antimicrob Chemother. 2017;72(5):1496-501.

118. Pamer EG. Resurrecting the intestinal microbiota to combat antibiotic-resistant pathogens. Science (New York, NY). 2016;352(6285):535-8.

119. Lee JR, Magruder M, Zhang L, et al. Gut microbiota dysbiosis and diarrhea in kidney transplant recipients. Am J Transplant Off J Am Soc Transplant Am Soc Transplant Surg. 2018.

120. Wu ZW, Ling ZX, Lu HF, Zuo J, Sheng JF, Zheng SS, et al. Changes of gut bacteria and immune parameters in liver transplant recipients. Hepatobiliary \& Pancreatic Diseases International: HBPD INT. 2012;11(1):40-50.

121. Herati RS, Blumberg EA. Losing ground: multidrug-resistant bacteria in solid-organ transplantation. Curr Opin Infect Dis. 2012;25(4):445-9.

122. Patolia S, Abate G, Patel N, Patolia S, Frey S. Risk factors and outcomes for multidrug-resistant Gram-negative bacilli bacteremia. Therapeutic Advances in Infectious Disease. 2018;5(1): $11-8$.

123. Kaleko M, Bristol JA, Hubert S, Parsley T, Widmer G, Tzipori S, et al. Development of SYN-004, an oral beta-lactamase treatment to protect the gut microbiome from antibiotic-mediated damage and prevent Clostridium difficile infection. Anaerobe. 2016;41: $58-67$.

124. Roberts T, Kokai-Kun JF, Coughlin O, Lopez BV, Whalen H, Bristol JA, et al. Tolerability and pharmacokinetics of SYN-004, an orally administered beta-lactamase for the prevention of Clostridium difficile-associated disease and antibiotic-associated diarrhea, in two phase 1 studies. Clinical Drug Investigation. 2016;36(9):725-34.

125. Bristol A, Hubert S, Hofmann F, Baer H. Formulation development of SYN-004 (ribaxamase) oral solid dosage form, a beta- lactamase to prevent intravenous antibiotic-associated dysbiosis of the colon. Int J Pharm. 2017;534(1-2):25-34.

126. Kokai-Kun JF, Roberts T, Coughlin O, et al. The oral betalactamase SYN-004 (Ribaxamase) degrades ceftriaxone excreted into the intestine in phase 2a clinical studies. Antimicrob Agents Chemother 2017;61(3). The drug investigated in the study presents an entirely novel approach to reducing development of resistance in the gastrointestinal tract for patients exposed to broadly acting antibiotics.

127. Wong WF, Santiago M. Microbial approaches for targeting antibiotic-resistant bacteria. Microb Biotechnol. 2017;10(5): 1047-53.

128. Aroniadis OC, Brandt LJ, Greenberg A, Borody T, Kelly CR, Mellow M, et al. Long-term follow-up study of fecal microbiota transplantation for severe and/or complicated Clostridium difficile infection: a multicenter experience. J Clin Gastroenterol. 2016;50(5):398-402.

129. Crum-Cianflone NF, Sullivan E, Ballon-Landa G. Fecal microbiota transplantation and successful resolution of multidrugresistant-organism colonization. J Clin Microbiol. 2015;53(6): 1986-9.

130. Mamo Y, Woodworth MH, Wang T, Dhere T, Durability KCS. Long-term clinical outcomes of fecal microbiota transplant treatment in patients with recurrent Clostridium difficile infection. Clinical Infectious Diseases: An Official Publication of the Infectious Diseases Society of America. 2018;66(11):1705-11.

131. Halpin AL, McDonald LC. Editorial commentary: the dawning of microbiome remediation for addressing antibiotic resistance. Clinical Infectious Diseases: An Official Publication of the Infectious Diseases Society of America. 2016;62(12):1487-8.

Publisher's Note Springer Nature remains neutral with regard to jurisdictional claims in published maps and institutional affiliations. 